\title{
A Novel Routing Control Technique for the Energy Hole in the Underwater Acoustic Distributed Network
}

\author{
Dong Xiao $\mathbb{D}^{1},{ }^{1}$ Min Zhao, ${ }^{2}$ Ning Jia, ${ }^{1}$ Tong-Rui Peng $\mathbb{D},{ }^{3}$ Yan Chen, ${ }^{1}$ and Li Ma ${ }^{1}$ \\ ${ }^{1}$ Institute of Acoustics, Chinese Academy of Sciences, Beijing 100190, China \\ ${ }^{2}$ Economic Research Institute, State Grid Jibei Electric Power Company Limited, Beijing 100038, China \\ ${ }^{3}$ College of Science, Shanghai Institute of Technology, Shanghai 201418, China \\ Correspondence should be addressed to Dong Xiao; xiaodong@mail.ioa.ac.cn and Tong-Rui Peng; tongrui2.peng@sit.edu.cn
}

Received 18 November 2020; Revised 11 January 2021; Accepted 27 January 2021; Published 18 February 2021

Academic Editor: Rui Wang

Copyright (C) 2021 Dong Xiao et al. This is an open access article distributed under the Creative Commons Attribution License, which permits unrestricted use, distribution, and reproduction in any medium, provided the original work is properly cited.

The energy hole is a severe problem for underwater acoustic distributed networks in that it affects the normal operations of the network and shortens the network's life span. To deal with this problem, a loop-free routing control technique is proposed in this paper. The classical shortest-path routing control method is used to generate multiple disjointed routing tables. The residual energy of the nodes and the changing information of the uplink/downlink matrix are added to the data frames for distribution. The source node specifies the routing path to transmit the data frames based on the changing information, and the relay nodes route the data frames strictly according to the routing path designated by the source node. Besides, the energy consumption of the relay node is saved by replying to the pseudo-ACK frame. Simulation experiments are implemented in four typical scenarios, and the results reflect that the proposed technique could extend the network's life span by approximately $10 \%$ when compared to other mature techniques. Besides, it has no other negative effects on the normal operations of the network.

\section{Introduction}

1.1. Background and Literature Review. The underwater acoustic distributed network which consisted of fixed sensor nodes is widely used for collecting data and exchanging information in the environment of underwater [1]. However, the energy holes are severely affecting the normal operations of the system and shortening the network's life span [2]. To avoid energy holes, a variety of excellent engineering methods are proposed, e.g., reducing hardware power consumption [3], decreasing software calculations, sleep-awaken strategies [4], optimizing and configuring battery power, adjusting node-deployment density [5], configuring topology [6], and other methods through balancing the energy consumption of nodes. The main design ideas are based on four assumptions, which are specified as follows:

(1) No auxiliary information or equipment is needed, and the cost function is designed to adjust the transmission power or routing path based on factors, such as residual energy of nodes, channel characteristics, and link quality [2, 7-9]

(2) The auxiliary information, such as the geographic location, the deployment depth, the azimuth of arrival, and distances between nodes, is used to adjust the transmission power or routing path [10-17]

(3) Machine learning methods (e.g., Q-learning and ant colony algorithm) are used [18-20]

(4) Mobile nodes are used as auxiliary equipment for data collection, and their movement trajectories and collection strategies are planned [21-26]

An arc-shaped hierarchical network composed of 1 central node and 50 static nodes is constructed in [7]. It selects the routing paths based on the efficiency of the path energy and the path lifetime. Besides, prior knowledge of the network topology is required. A congestion control and load balancing technique for cluster-based underwater acoustic sensor networks is proposed in [8], but the route hops or paths are not mentioned. A routing strategy that leads to an 
even energy depletion among all sensors in the network and consequently an improved network life span is proposed in [2], but the network is assumed to be arranged as a star topology. A dynamic routing control method is presented in [9], but the route paths are controlled and distributed only by the sink node, which consumes considerable energy. A routing algorithm based on link quality is proposed in [10], which reduces retransmission times, but requires the geographic location information of the node as support. Nodes' one-hop neighbour is redefined based on signal attenuation, and a distributed energy-efficient routing protocol for UASNs is designed in [11], but it assumes each node knows its neighbours' positions. An energy-balancing and delayrestricted routing protocol is proposed in [12], but the location, residual energy, and load of its neighbours must be aware of by every node. A new clustering algorithm based on the low-energy adaptive clustering hierarchy (LEACH) protocol is proposed in [13], but the cluster head's position is needed. An improved protocol called energy-balanced pressure routing (EBPR) is proposed in [14], but the depth information of each node and periodical broadcast are needed. An improved reliability and energy-balanced routing algorithm (REBAR) is proposed in [15], but distances between source nodes and sink nodes are needed. A detailed description of the ad hoc underwater acoustic network protocol is presented in [16], which is an improved ad hoc on-demand distance vector (AODV) routing control method, and distance information is needed. An energyefficient routing scheme is presented for underwater acoustic sensor networks (UW-ASN) in [17], which is based on the directed diffusion routing scheme. An adaptive, energy-efficient, and lifetime-aware routing protocol based on reinforcement learning, QELAR, is proposed and simulated in $[18,19]$, but considerable energy is consumed by periodical broadcast. A fair energy usage routing algorithm, which uses the ant colony optimization algorithm (ACO) to balance the network energy, is proposed in [20], but it is designed for wireless sensor networks only.

In this study, the nodes are deployed randomly so that some essential information, e.g., neighbour information, depth information of other nodes, and geographical location information, is difficult to obtain, and no mobile node is deployed. Therefore, only the first and the third types of assumptions are considered. The first and third types of assumptions require routing control for transmitting data, and table-driven routing [5, 27] and on-demanding routing $[28,29]$ are two main routing control methods. Currently, the on-demand routing control method has been reported in most published reports. On the one hand, considering that the fixed underwater acoustic distributed network nodes carry limited energy and are difficult to move after deployment, the routing changes are relatively rare, and there is no need to search for routing paths repeatedly. On the other hand, most load balancing methods do not focus on routing loops that are prone to occur when routing paths change.

1.2. The Motivation of This Paper. It is found that when geographic location and topology information is lacking, routing loops are likely to occur when searching for the routing path to achieve load balancing. Repeated route searching and routing loops both lead to rapid node energy consumption, shortened network life, large channel resource occupation, and increased end-to-end delays of data packets. A few methods use loop detection to break routing loops, but they cannot fundamentally eliminate routing loops. This paper concentrates on how to delay the appearing time of the energy hole in the underwater acoustic distributed network as late as possible, and that is to save the energy of the nodes on critical routing paths and eliminate routing loops while searching for the routing path to balance the load.

\subsection{Contributions of This Paper. There are four contributions} of this paper.

A novel loop-free routing control technique for the energy hole in the underwater acoustic distributed network is proposed to delay the appearance time of the energy hole (the first failed node) without negative effects on the normal operations.

To avoid the routing loop problem, the routing path of the data frame is designated by its source node, and the relay nodes will route the data frame strictly according to the routing path designated by the source node.

The link quality and the residual energy of the nodes are used by the source node to select the routing path, and the data load and the energy consumption are balanced.

The energy consumption is saved by replying pseudoACK frames by relay nodes.

1.4. Paper Organization. The rest of the paper is organized as follows: Section 2 describes the problem formulation of the energy hole in underwater acoustic distributed networks. Section 3 presents a detailed introduction of the proposed technique. Section 4 provides a detailed description of the simulation experiments and analyses the results to demonstrate the advantages of the proposed technique. Section 5 concludes the paper.

\section{Problem Formulation}

For underwater acoustic distributed networks, extended propagation delays, severe attenuations, narrow available communication bandwidth, low communication rates, and complicated underwater acoustic environments will increase the consumption of the transmission energy. Owing to premature energy exhaustion, the energy holes, which refer to failure nodes located on critical routing paths, become challenging problems, and they affect the network's normal operation and shorten the life span.

To solve the energy hole problems, the load-balanced methods, which extend the life of the network by balancing the energy consumption of each node, are widely used. However, once a routing loop is formed, the data frames will be transmitted within the network repeatedly and cannot reach the destination node. Consequently, the energy of the node is consumed quicker and the limited channel resource is occupied a lot, which damage the performance of the loadbalanced methods. 
In this study, a novel loop-free route control technique is proposed for solving the routing loop problem. The classical shortest-path routing control method is used to generate multiple disjointed routing tables. The residual energy of the nodes and the changing information of the uplink/downlink matrix are added to the data frames for distribution. The source node specifies the routing path to transmit the data frame based on the changing information, while relay nodes route the data frame strictly according to the routing path designated by the source node. Besides, the energy consumption of the relay node is saved by replying the pseudoACK frame.

\section{Routing Control Technique for the Energy Hole}

3.1. Generation of the Routing Table. The procedures of generating the routing table can be explained as follows:

(1) Assume there are totally $T$ nodes in the network and at least two other nodes in the communication range of each node, which means that each node has at least one backup routing table.

(2) A unique address $t$, where $t \in[0, T)$, is initialized for each node.

(3) A link matrix, $Q$, and a residual energy table, $E$, must be built through the self-organization process (see Appendix) for each node, wherein link matrix, $Q$, has $T$ rows and $T$ columns, with the element, $q_{i j}$, in row $i$ and column $j$, representing the link quality from node $i$ to node $j$, and $q_{i j} \in[0, Q], i, j \in[0, T)$. The residual energy table, $E$, has $T$ rows and one column, with the element, $e_{i}$, of the $i$-th term being the residual energy of node $i$, where $e_{i} \in[0, E]$.

(4) Routing tables are created according to $Q$ and $E$.

(5) A routing index table, $S$, is created after all routing tables have been calculated. $S$ contains $T$ rows and one column, where the $i$-th element, $s_{i}$, indicates that the $i$-th routing path in group $t$, recorded in the $s_{i}$-th routing table, is selected as the routing path from the local node to node $i$.

3.2. Data Transmission. Figure 1 presents the format of the data frame. The change information of the link matrix includes row/column number, uplink/downlink, and change results, which are used to communicate the uplink/downlink changes between the local node and others. If the destination node address of the data frame is $d s t$, it is transmitted according to the $d s t$-th routing path of group $t$ in the $s_{d s t} t^{\text {th }}$ routing table. After transmission, the information and transmission time of the data frame are recorded and enter the ACK waiting state. If ACK waiting timer expires, the data frame will be retransmitted, and the transmission record will be updated. If the retransmission limit is exceeded, the data frame will be put into the tail of transmitting queue. The flowchart of the node-transmitting terminal is shown in Figure 2.
3.3. Data Reception. Figure 3 presents the flowchart of the node-receiving terminal, and data processing can only be performed when the CRC checksum is correct. Each node follows this flowchart to process the received data frame and update the transmitting information table.

3.3.1. Selection of the Routing Table. Update the link matrix, $Q$, and the residual energy table, $E$, according to the received data frame, and note the node address with the smallest residual energy in the residual energy table as $\mathrm{em}$. Then, according to equation (1), the routing cost, $t m p_{b}$, from local node $t$ in the entire routing table to any other node, $j$, is calculated:

$$
t m p_{b}= \begin{cases}0, & j=t, \\ \infty, & j \neq t \text { 且 } r h_{b t j}=0, \\ \sum_{k=1}^{r h_{b t j} \frac{\Delta c h_{b k}+\Delta e n_{b k}}{r h_{b t j}},} & j \neq t \text { 且 } r h_{b t j} \neq 0,\end{cases}
$$

wherein

$$
\begin{aligned}
& \Delta e n_{b k}= \begin{cases}0, & r r_{b t j k}=G, \\
W E^{2} \times\left(E-e_{j k}\right), & r r_{b t j k}=e m, \\
W E \times\left(E-e_{j k}\right), & \text { else, }\end{cases} \\
& \Delta c h_{b k}= \begin{cases}0, & r r_{b t j k}=G, \\
W Q^{2} \times\left(Q-q_{j k}\right), & r r_{b t j k} \neq G q_{j k}=0, \\
W Q \times\left(Q-q_{j k}\right), & r r_{b t j k} \neq G q_{j k} \neq 0 .\end{cases}
\end{aligned}
$$

$G$ means broadcast. When $j=t$, the local loop path of the local node is given, and no calculation is needed. $r h_{b t j}$ is the hop count required by the local node to reach node $j$ in the $b$ th routing table. The hop count is used as the denominator to allow some routing paths having a large number of hop counts to be selected. $\Delta e n_{b k}$ is the residual energy cost, and $\Delta c h_{b k}$ is the link quality cost. $r r_{b t j k}$ is the address of the $k$-th hop node in the $b$-th routing table from the local node to node $j, b \in[0, B]$, and $B$ is the routing table having lowest selection cost, and the sequence number of this routing table is assigned to $s_{j}$, as shown in the following:

$$
s_{j}=\underset{b \in[0, B)}{\arg \min }\left\{t m p_{b}\right\} .
$$

When the data frame sent by the source node does not receive the ACK frame returned by the destination node within a limited time, the routing index table, $S$, is updated according to the above process and retransmitted.

3.3.2. Update of the Link Matrix. When the data frame transmitted or relayed by this node is correctly responded to by the correct next hop node within the limited time, the uplink/downlink matrix of the node is updated according to equations (4) and (5): 


\begin{tabular}{|c|c|c|c|c|c|c|c|c|c|c|c|}
\hline Тур & Src & Cur & Nxt & Dst & Hop & Eng & $\mathrm{U} / \mathrm{D}$ & $\mathrm{R} / \mathrm{C}$ & Chg & No. & CRC \\
\hline
\end{tabular}

Figure 1: Format of the data frame. ACK frame does not include the Dat segment.

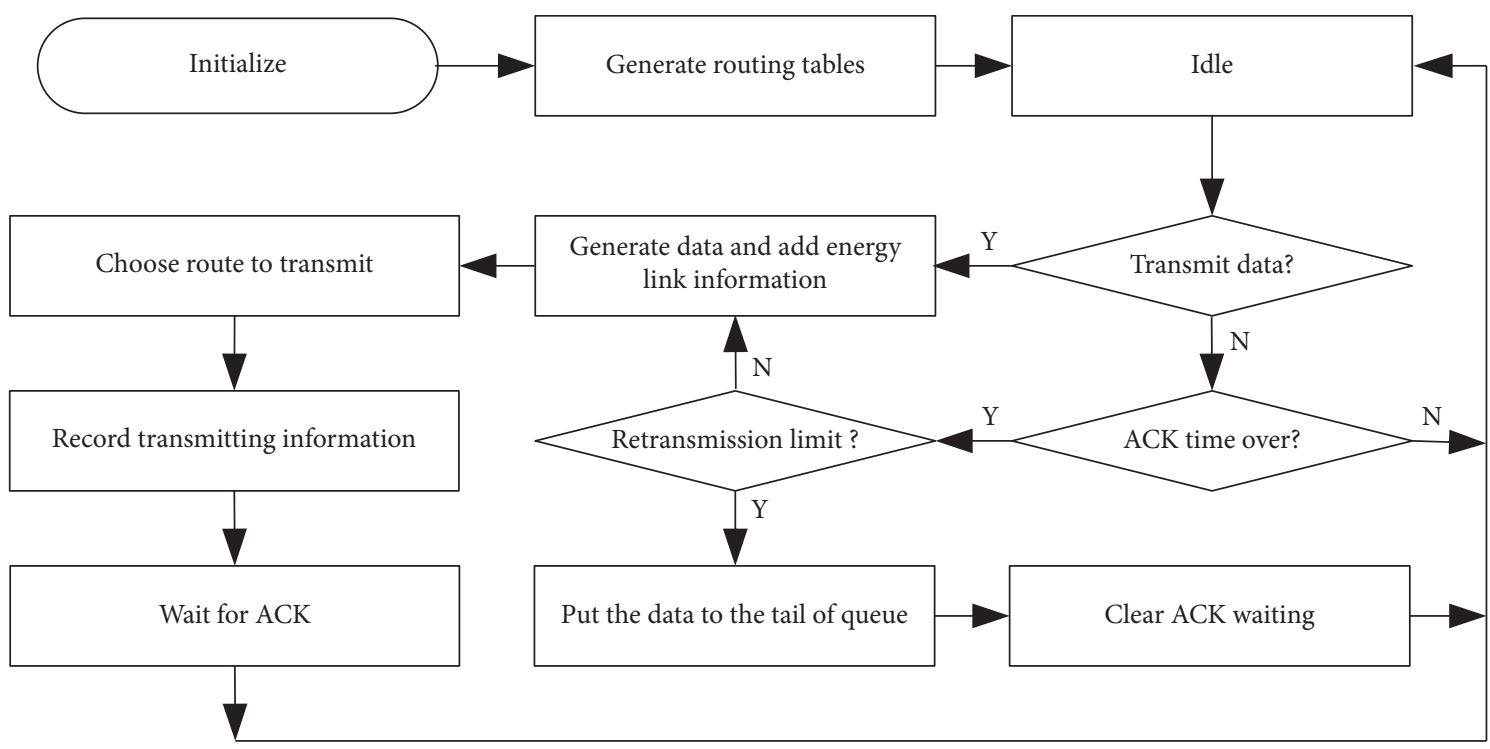

Figure 2: Flowchart of the transmitting terminal.

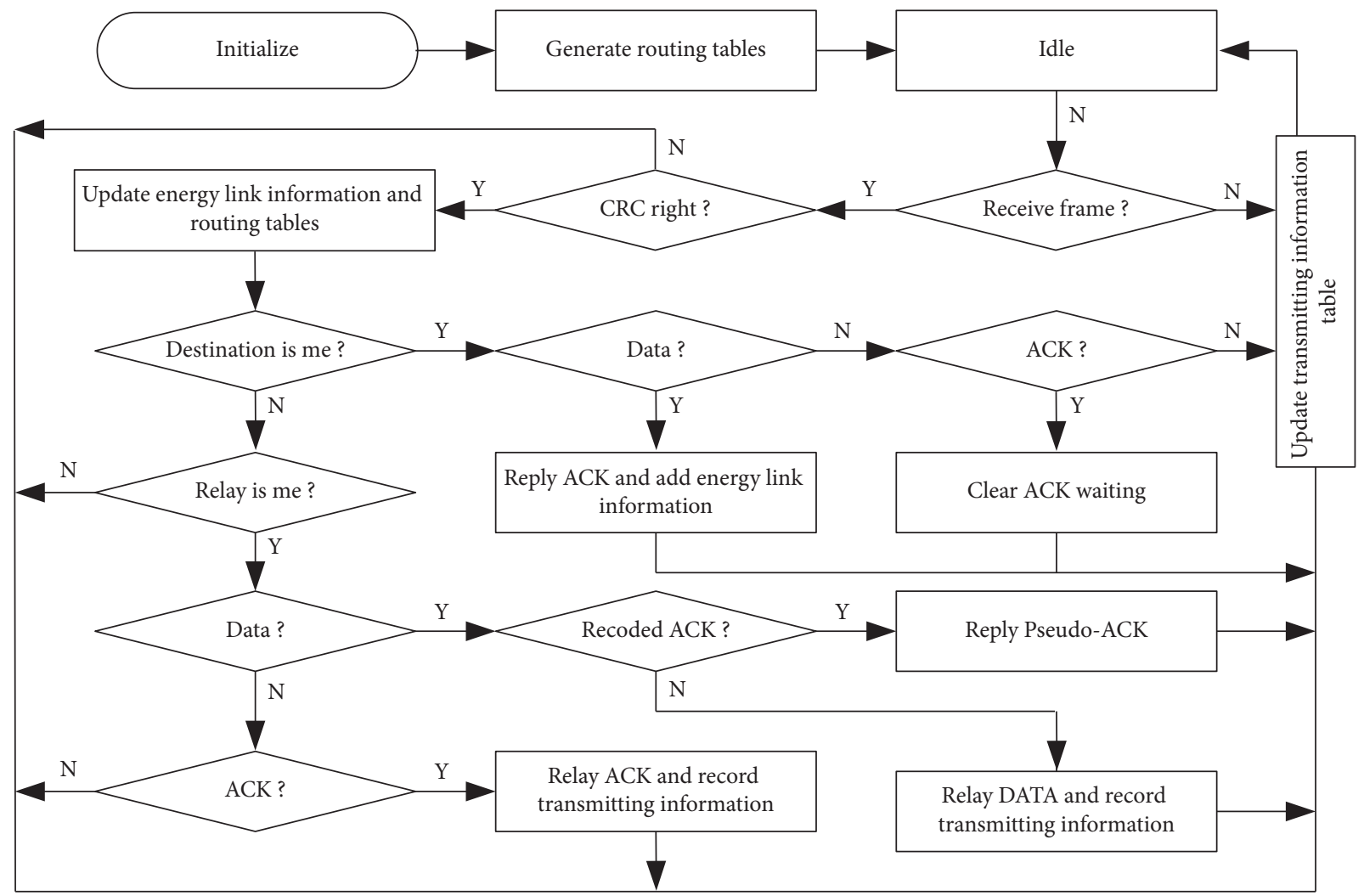

FIGURE 3: Flowchart of the receiving terminal. 


$$
\begin{aligned}
& q_{t c u r}= \begin{cases}Q, & q_{t c u r}=Q, \\
q_{t c u r}+1, & \text { else, }\end{cases} \\
& q_{\text {curt }}= \begin{cases}Q, & q_{\text {curt }}=Q, \\
q_{\text {cur } t}+1, & \text { else. }\end{cases}
\end{aligned}
$$

If the correct response is not received within the limited time, the uplink/downlink matrix of the node is updated according to equations (6) and (7), respectively:

$$
\begin{aligned}
& q_{t \text { cur }}= \begin{cases}0, & q_{t c u r}=0, \\
q_{t c u r}-1, & \text { else, }\end{cases} \\
& q_{\text {curt }}= \begin{cases}0, & q_{\text {curt }}=0, \\
q_{c u r t}-1, & \text { else. }\end{cases}
\end{aligned}
$$

In equations (4)-(7), cur represents the next hop address. The correct response includes two cases. When the next hop address of the frame is the relay node, the frame should be relayed. When the next hop node is the destination node of the data frame, the ACK frame should be replied. After the update is completed, when the change of an element in the link matrix exceeds a certain threshold, the change is assigned to the next data frame sent by the node. When the data frame sent by the source node does not receive the ACK frame replied by the destination node within a limited time, the corresponding elements in the uplink/downlink matrix are valued according to equations (6) and (7), respectively.

3.3.3. Data Relay. When a node receives a frame from another node, if the next hop address in the frame is the address of itself, it must relay the frame. Before relaying a data frame, the node must check its own transmission record. If it is found that an ACK frame corresponding to the data frame has been transmitted, it pretends that it is the destination node of the data frame and replies a pseudoACK frame to the source node. If there is no transmission record corresponding to the data frame that needs to be relayed before relaying a DATA frame, the routing path from the source node of the data frame to its destination node in all routing tables must be found. Then, according to the path, the current address in the required relay data frame is changed to its own node number, and the next hop address is changed to the next-hop node number on the path. After the hop count is increased by one, the content redundancy check is recalculated and transmitted.

\section{Results and Discussion}

A half-duplex underwater acoustic communication method is designed using OPNET software, a tool used to simulate the behaviour and performance of different types of networks, and the underwater acoustic channel is modelled. Four typical node-deployment scenarios are simulated, and simulation results are compared with those using a traditional shortest-path route control method.
4.1. Modelling and Design of Half-Duplex Underwater Acoustic Communication. The wireless link is divided into 14 pipeline model stages for modelling in OPNET. In the node model, the centre frequency, the bandwidth, the modulation method, the transmission rate, and the errorcorrection coding of both transmitter and receiver are set. In OPNET, the wireless link adopts the full-duplex mode by default. However, at present, most underwater acoustic communications can only use half-duplex.

During the simulation, the underwater acoustic communication parameters are set to BPSK (binary phase-shift keying) modulation in the half-duplex mode. The centre frequency is $8 \mathrm{kHz}$, the bandwidth is $4 \mathrm{kHz}$, the communication rate is $1 \mathrm{kbps}$, the effective distance is $5 \mathrm{~km}$, the transmission power is $20 \mathrm{~W}$, the standby (including receiving) power is $0.1 \mathrm{~W}$, and the battery capacity of each node is $16,000 \mathrm{~J}$. Additionally, the master node is powered by shore-based power, excluding energy consumption. In the underwater acoustic communication process, the following pipeline model stages need attention.

In stage 6 , the underwater wave propagation speed $(1,500 \mathrm{~m} / \mathrm{s})$ differs from the default electromagnetic-wave propagation speed and must be modified.

In stage 8 , the code must be rewritten based on the underwater acoustic propagation attenuation model to calculate the received power. The mesoscale empirical formula in the classic Marsh-Schulkin model is used in the simulation process, and the formula is

$$
T L=15 \log _{10} R+\alpha R+\alpha_{t}\left(\frac{R}{H}-1\right)+5 \log _{10} H+60-k_{L},
$$

where $H=[(L+D) / 3]^{0.5}$ is the span $(\mathrm{km}), L$ is the depth of the mixed layer $(\mathrm{m}), D$ is the depth of the ocean $(\mathrm{m}), R$ is the horizontal distance $(\mathrm{km}), \alpha$ is the absorption coefficient $(\mathrm{d} B /$ $\mathrm{km}), \alpha_{t}$ is the effective attenuation coefficient in shallow water $(\mathrm{d} B$, reflection loss per sea surface-bottom reflection, which is related to frequency, sea conditions, and bottom quality), and $k_{L}$ is the near-field anomaly correction $(\mathrm{d} B$, related to frequency and bottom quality).

$$
\begin{aligned}
& \alpha_{t}=2.0+0.25 \times(f-2.0), \\
& \alpha=8.686 \times 10^{3} \times\left[\frac{S A f_{T} f^{2}}{f_{T}^{2}+f^{2}}+\frac{B f^{2}}{f_{T}}\right]\left(1-6.54 \times 10^{-4} P\right) .
\end{aligned}
$$

The unit of $\alpha$ is $\mathrm{d} B / \mathrm{km}, S$ is thousandth of salinity (usually, $35 \%$ ) $, A=2.34 \times 10^{-6}, B=3.38 \times 10^{-6}, f$ is the acoustic frequency $(\mathrm{kHz}), P$ is the static pressure (atmospheric pressure), and $f_{T}$ is the relaxation frequency $(\mathrm{kHz})$.

$$
f_{T}=21.9 \times 10^{(6-(1520 /(T+273)))} .
$$

In actual underwater acoustic communications, when collisions occur between multiple received signals, they cannot be received correctly. Therefore, the influence of noise crosstalk between signals is amplified during the noise crosstalk of stage 9 . 


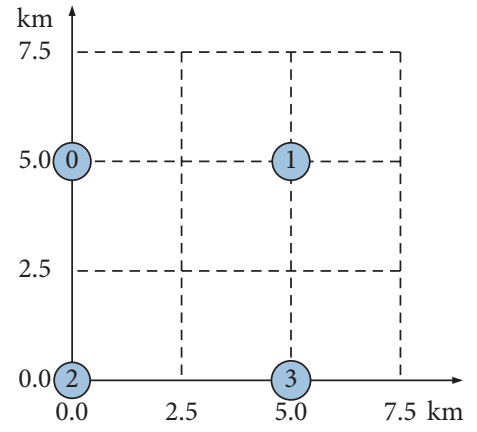

(a)

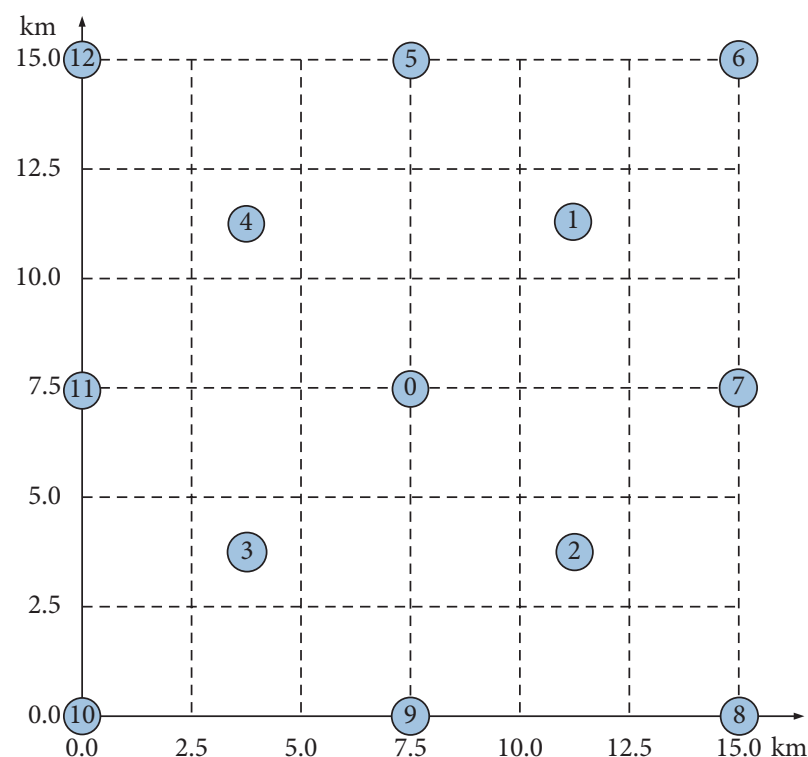

(c)

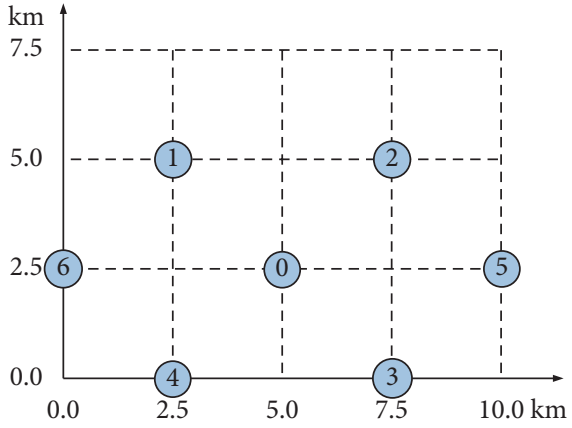

(b)

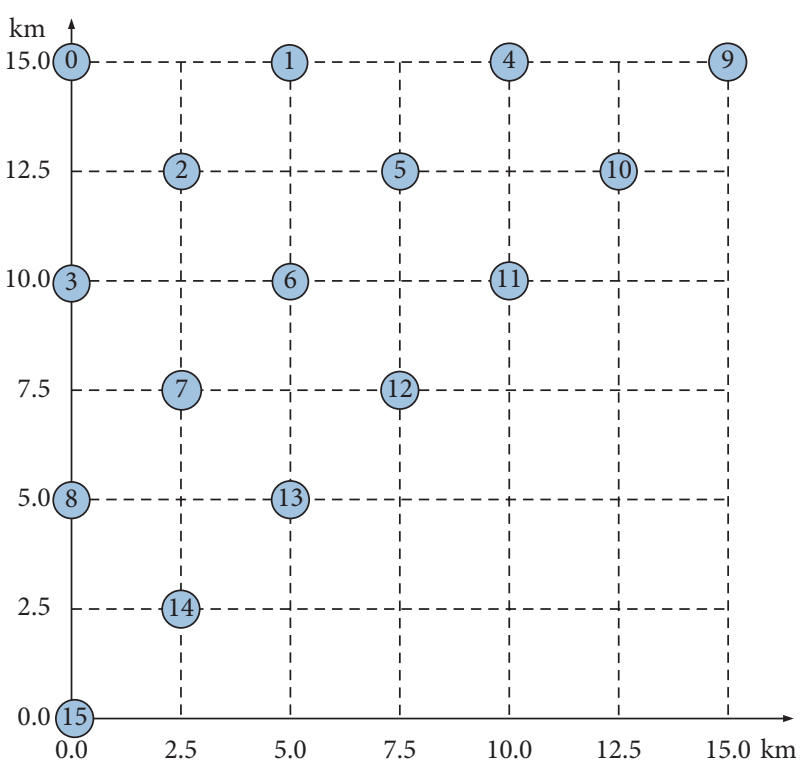

(d)

Figure 4: Simulation cases. (a) Case of 4 nodes. (b) Case of 7 nodes. (c) Case of 13 nodes. (d) Case of 16 nodes.

In stage 10, the ocean background noise level should be calculated, and a classic empirical formula should be adopted in the simulation [30]. It is divided into four categories for calculation, as shown in the following:

$$
N L_{B}=N L+10 \log _{10} B,
$$

where

$$
\begin{aligned}
N L & =10 \log _{10}\left(\sum_{i=1}^{4} 10^{N L_{i} / 10}\right), \\
N L_{1} & =17-30 \log _{10} f, \\
N L_{2} & =40+20(V D-0.5)+26 \log _{10} f-60 \log _{10}(f+0.03), \\
N L_{3} & =50+7.5 w^{0.5}+20 \log _{10} f-40 \log _{10}(f+0.4), \\
N L_{4} & =-15+20 \log _{10} f .
\end{aligned}
$$

In the formula, $V D$ represents the vessel density, and $V D \in[0,1] . B$ is the signal bandwidth. $f$ is the centre frequency of the signal, and the unit is HZ. $w$ is the wind speed, and the unit is $\mathrm{m} / \mathrm{s}$.

By adjusting parameters, such as transmission power, in the 11th stage, the bit error rates without collision are about $10^{-3}$, and the bit error rates with collision are in the order of $10^{-1}$.

4.2. Results' Analysis. Figure 4 presents the simulation results for the four typical node-deployment situations using the traditional shortest-path routing control method.

Considering the case of node 4, the lengths of each segment of the data frame are data type ( 4 bits), source address ( 4 bits), current address ( 4 bits), next hop address (4 bits), destination address ( 4 bits), hop count (4 bits), residual energy of the source ( 4 bits), change information of the link matrix ( 4 bits), frame number ( 8 bits), data segment (256 bits), and checking ( 8 bits). The ratio of useful information (data segment) is $84.2 \%$. A link matrix, $Q$, and a residual energy table, $E$, are established for each node through a network self-organization method, wherein $Q=3$, $E=15$, and 
TABle 1: Route table of each node.

\begin{tabular}{|c|c|c|c|c|c|c|c|}
\hline & Source & Destination & Hop 0 & Hop 1 & Hop 2 & Hop 3 & Hop count \\
\hline \multirow{16}{*}{ Route table 0} & \multirow{4}{*}{ Node 0} & Node 0 & 0 & G & G & G & 0 \\
\hline & & Node 1 & 0 & 1 & G & G & 1 \\
\hline & & Node 2 & 0 & 2 & G & G & 1 \\
\hline & & Node 3 & 0 & 2 & 3 & G & 2 \\
\hline & \multirow{5}{*}{ Node 1} & Node 0 & 1 & 0 & G & G & 1 \\
\hline & & Node 1 & 1 & G & G & G & 0 \\
\hline & & Node 2 & 1 & 3 & 2 & G & 2 \\
\hline & & Node 3 & 1 & 3 & G & G & 1 \\
\hline & & Node 0 & 2 & 0 & G & G & 1 \\
\hline & \multirow{3}{*}{ Node 2} & Node 1 & 2 & 3 & 1 & G & 2 \\
\hline & & Node 2 & 2 & G & G & G & 0 \\
\hline & & Node 3 & 2 & 3 & G & G & 1 \\
\hline & \multirow{4}{*}{ Node 3} & Node 0 & 3 & 2 & 0 & G & 2 \\
\hline & & Node 1 & 3 & 1 & G & G & 1 \\
\hline & & Node 2 & 3 & 2 & G & G & 1 \\
\hline & & Node 3 & 3 & G & G & G & 0 \\
\hline \multirow{16}{*}{ Route table 1} & \multirow{4}{*}{ Node 0} & Node 0 & 0 & G & G & G & 0 \\
\hline & & Node 1 & 0 & 1 & G & G & 1 \\
\hline & & Node 2 & 0 & 2 & G & G & 1 \\
\hline & & Node 3 & 0 & 2 & 3 & G & 2 \\
\hline & \multirow{5}{*}{ Node 1} & Node 0 & 1 & 0 & G & G & 1 \\
\hline & & Node 1 & 1 & G & G & G & 0 \\
\hline & & Node 2 & 1 & 3 & 2 & G & 2 \\
\hline & & Node 3 & 1 & 3 & G & G & 1 \\
\hline & & Node 0 & 2 & 0 & G & G & 1 \\
\hline & \multirow{3}{*}{ Node 2} & Node 1 & 2 & 3 & 1 & G & 2 \\
\hline & & Node 2 & 2 & G & $\mathrm{G}$ & G & 0 \\
\hline & & Node 3 & 2 & 3 & G & G & 1 \\
\hline & \multirow{4}{*}{ Node 3} & Node 0 & 3 & 2 & 0 & G & 2 \\
\hline & & Node 1 & 3 & 1 & G & G & 1 \\
\hline & & Node 2 & 3 & 2 & G & G & 1 \\
\hline & & Node 3 & 3 & G & G & G & 0 \\
\hline
\end{tabular}

TABLe 2: Comparison results of different techniques (in terms of network lifetime).

\begin{tabular}{lccccccccccc}
\hline \multirow{4}{*}{4 nodes } & $1 \mathrm{~min}$ & $2 \mathrm{~min}$ & $3 \mathrm{~min}$ & $4 \mathrm{~min}$ & $5 \mathrm{~min}$ & $6 \mathrm{~min}$ & $7 \mathrm{~min}$ & $8 \mathrm{~min}$ & $9 \mathrm{~min}$ & $10 \mathrm{~min}$ \\
\hline \multirow{4}{*}{7 nodes } & Traditional & 451.8 & 703.4 & 909.6 & 1080.6 & 1215.6 & 1326.7 & 1428.5 & 1488.6 & 1584.5 & 1630.6 \\
& Greedy & 518.6 & 830.5 & 1050.6 & 1232.6 & 1395.5 & 1470.6 & 1575.5 & 1680.6 & 1755.6 & 1810.6 \\
& Proposed & 558.5 & 878.6 & 1101.8 & 1264.5 & 1380.6 & 1506.8 & 1617.7 & 1688.6 & 1755.7 & 1800.6 \\
\hline \multirow{3}{*}{13 nodes } & Traditional & 693.6 & 1030.6 & 1302.6 & 1468.6 & 1595.6 & 1686.6 & 1778.8 & 1872.6 & 1926.5 & 1930.8 \\
& Greedy & 441.7 & 670.6 & 876.5 & 1016.5 & 1170.5 & 1248.7 & 1400.5 & 1464.5 & 1557.7 & 1580.7 \\
& Proposed & 841.5 & 1222.6 & 1479.8 & 1628.6 & 1760.6 & 1836.8 & 1911.7 & 1976.7 & 2034.6 & 2060.6 \\
\hline \multirow{3}{*}{16 nodes } & Traditional & 580.8 & 962.6 & 1185.5 & 1336.6 & 1480.6 & 1596.6 & 1652.8 & 1712.5 & 1809.6 & 1860.6 \\
& Greedy & 451.8 & 844.6 & 1132.5 & 1238.6 & 1396.7 & 1435.0 & 1561.8 & 1672.6 & 1737.6 & 1810.6 \\
& Proposed & 694.6 & 1080.8 & 1326.6 & 1484.7 & 1615.6 & 1723.9 & 1778.7 & 1856.8 & 1872.6 & 1960.7 \\
\hline & Traditional & 687.6 & 1080.6 & 1338.6 & 1488.6 & 1640.6 & 1698.6 & 1806.6 & 1840.8 & 1908.8 & 1950.6 \\
& Greedy & 792.0 & 1083.7 & 13447 & 1456.7 & 1590.9 & 1740.6 & 1778.5 & 1752.6 & 1881.6 & 1951.5 \\
& Proposed & 958.6 & 1382.6 & 1608.6 & 1784.6 & 1880.6 & 1950.7 & 2030.6 & 2088.6 & 2016.6 & 2100.6 \\
\hline
\end{tabular}




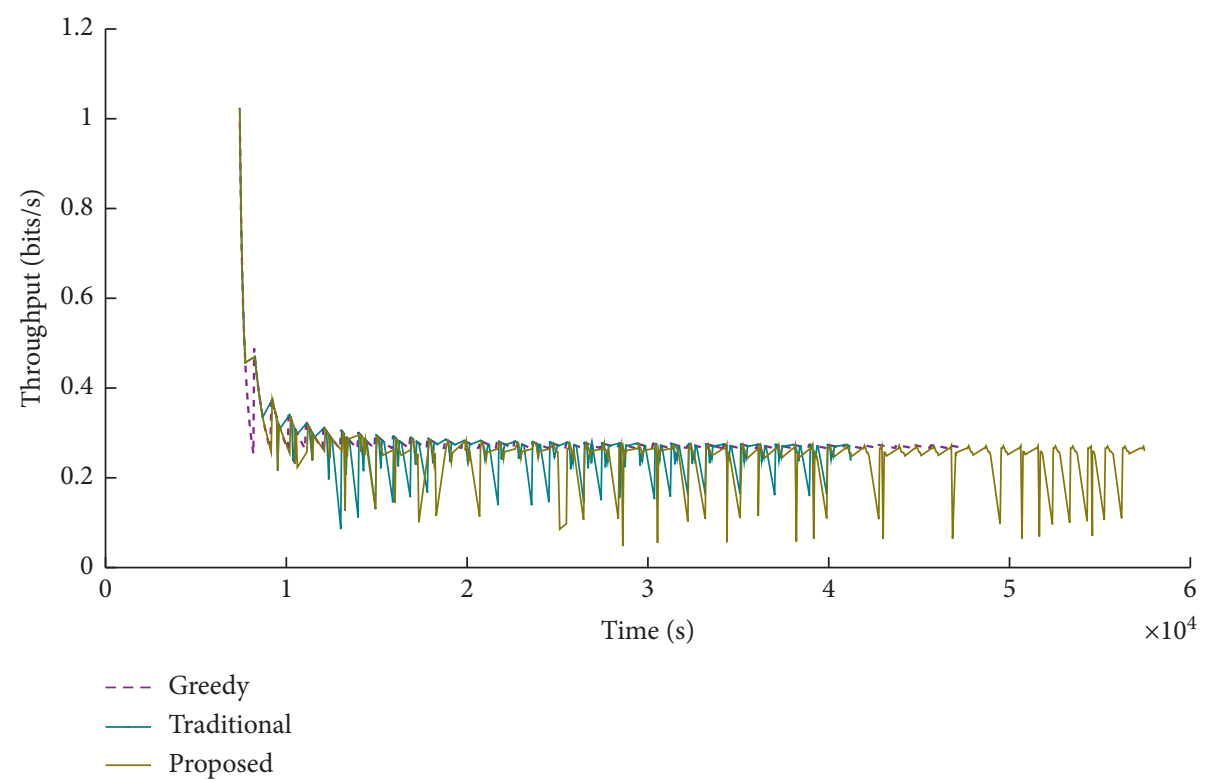

(a)

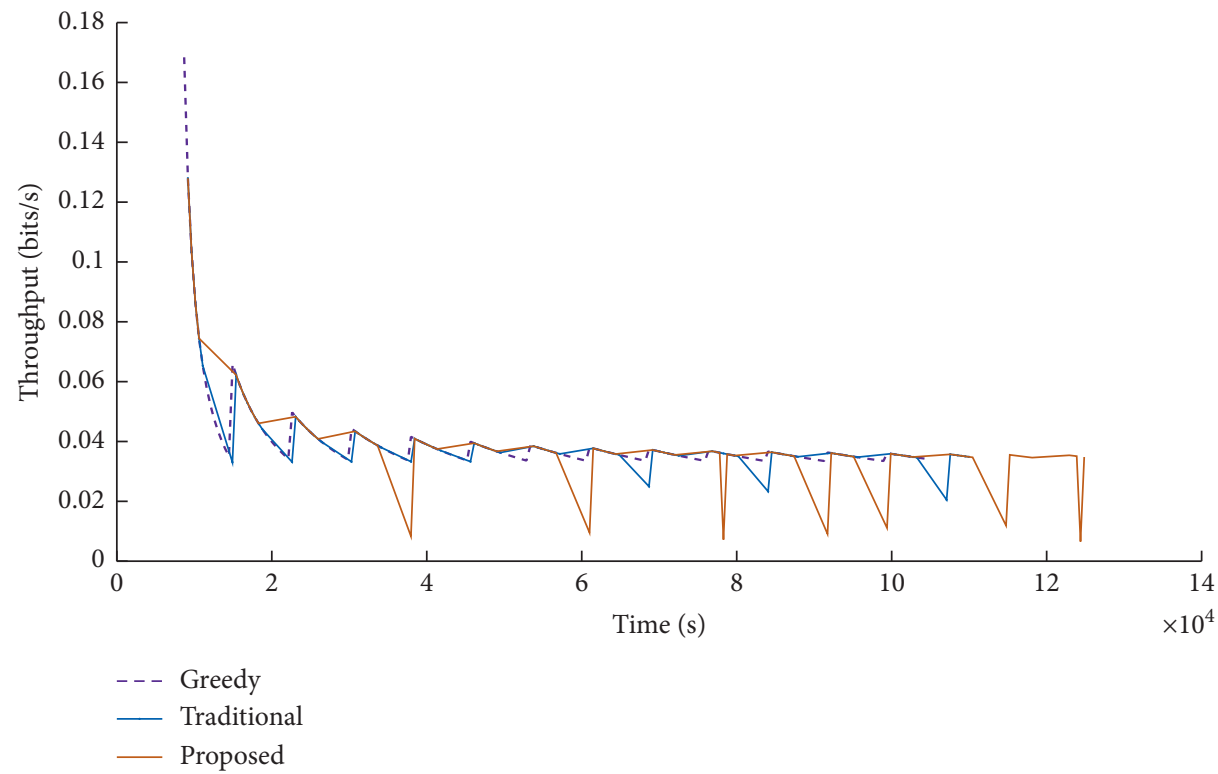

(b)

Figure 5: Comparison of the network throughput among the traditional technique, greedy technique, and proposed technique. (a) Transmission interval 1 minute. (b) Transmission interval 8 minutes. Traditional technique does not use load-balancing algorithms, and its lifetime is short than the proposed technique.

$$
\begin{aligned}
& Q=\left[\begin{array}{llll}
0 & 3 & 3 & 0 \\
3 & 0 & 0 & 3 \\
3 & 0 & 0 & 3 \\
0 & 3 & 3 & 0
\end{array}\right], \\
& E=\left[\begin{array}{llll}
15 & 15 & 15 & 15
\end{array}\right] .
\end{aligned}
$$

The routing table of each node is shown in Table 1, and G stands for "broadcast."
The transmission interval of the data frame in each case can be divided into 10 levels: $1,2,3,4,5,6,7,8,9$, and 10 minutes. Network lifetime in this paper is defined as the lifetime of the first exhausted node for the traditional technique, greedy technique, and proposed technique under different node-deployment conditions and different data frame intervals.

In Table 2, the comparisons of the network lifetime of the traditional technique, greedy technique, and proposed technique under different node-deployment conditions and different data frame intervals are listed, and the time unit is minute. Overall, compared with the traditional technique 
which does not use load-balancing route control, the proposed technique can extend the network life by about $10 \%$ on average. And the greedy technique which uses the greedy algorithm for load-balancing route control performs better than the traditional technique when no route loop is shaped during the simulation. If any route loop is shaped, the performance of the technique related becomes the worst.

The proposed technique extends the network life. Moreover, its network throughput is basically the same as the traditional technique. Take the case of 16 nodes in Figure 4(d) with data frame intervals of 1 minute and 8 minutes as an example. The network throughput comparisons between the traditional technique, greedy technique, and proposed technique are shown in Figure 5. The network throughput shown in the figures is the net throughput. Thus, only the number of bits contained in the data segment in the data frame is calculated. It can be seen from both figures that the throughput of the proposed technique is basically the same as that of the traditional technique and greedy technique.

\section{Conclusions}

In this study, a loop-free routing control technique is proposed to solve the problem of energy holes in underwater acoustic distributed networks. Both link qualities and residual energies of the nodes are used for balancing the data load. All nodes are learned through the same routing tables during the period of self-organization, and the source node specifies the routing path, and then the relay nodes follow it to route the data frames strictly. Besides, the pseudo-ACK frame is replied by the relay node to save energy consumption. Simulation experiments are implemented in four typical scenarios, and the results show that the proposed technique extends the network life by approximately $10 \%$ while ensuring the network throughput.

\section{Appendix}

The self-organization process is explained in the authors' previously published papers [31, 32]. Here, the authors provide a brief summary as follows:

(1) It is supposed that the network contains $T$ nodes. Each node has an empty link matrix, $Q$, and residual energy table, $E$, before deployment.

(2) After deployment of all nodes, each node sends neighbour-finding frames one by one for several turns. When other nodes receive the neighbourfinding frames, update their link matrix, $Q$, according to equation 5 , and set the corresponding element $e_{\text {cur }}$ in the energy table, $E$, to E. After several turns of neighbour finding, each node gets its own column of link matrix, $Q$, filled with some nonzero elements.

(3) Each node broadcasts the fusing frame just once, which contains its own column of link matrix, $Q$. When other nodes receive the fusing frame, fill the corresponding column of their own link matrices, $Q$, and broadcast the fusing frame again.
(4) Each node checks its link matrix, Q. If there is any empty column, send an inquiring frame to its neighbours to ask for the empty column. When the neighbours receive the inquiring frame, every neighbour checks its corresponding column of the link matrix, $Q$. If the corresponding column is not empty, the neighbour node will send the replying frame to answer the node who sends the inquiring frame. Each node takes the inquiring-replying for several turns until all nodes have the same link matrix, $Q$, and the same residual energy table, $E$, after completing self-organization.

(5) According to equation A1, each node calculates the elements of the routing matrix, $R$ :

$$
r_{i j}= \begin{cases}\infty, & q_{i j} \times e_{i}=0, \\ W Q \times\left(Q-q_{i j}\right)+W E \times\left(E-e_{i}\right), & q_{i j} \times e_{i} \neq 0 .\end{cases}
$$

$W Q$ and $W E$ are the weights of link quality and residual energy, respectively. According to the routing matrix, $R$, the related routing algorithm (e.g., Dijkstra) is used to calculate the routing table and the backup routing tables. When the backup routing table is being calculated, it is necessary to bypass the nodes that the previous route has passed in order to ensure that there are no overlapping nodes between the routing tables. Each routing table contains $T$ groups, and each group contains $T$ entries. Each entry in the routing table should contain information, such as the destination address, the next hop address to which the destination node should be delivered, and the hop count required to reach the destination node. For the unreachable node, the next hop address is assigned to "broadcast," and the hop count is assigned to "0."

\section{Data Availability}

Almost all the data are presented in the tables of this paper. If there is a need for any other information, the corresponding author may be contacted by e-mail.

\section{Conflicts of Interest}

The authors declare that there are no conflicts of interest regarding the publication of this paper.

\section{Acknowledgments}

This work was supported in part by the Youth Innovation Promotion Association, Chinese Academy of Sciences (no. 2020024) and the Institute of Acoustics Chinese Academy of Sciences Young Elite Researcher Project (no. QNYC201702).

\section{References}

[1] R. Thangarajan, T. Siva, and R. Boopalachakaravarthy, "Adaptive energy efficient routing protocol with extended lifetime in underwater sensor networks," in Proceedings of the ICCSNT, pp. 322-326, Dalian, China, October 2013. 
[2] F. Bouabdallah, C. Zidi, and R. Boutaba, "Joint routing and energy management in underwater acoustic sensor networks," IEEE Transactions on Network and Service Management, vol. 14, no. 2, pp. 456-471, 2017.

[3] W. Elmannai, K. Elleithy, A. Shrestha et al., "A new algorithm based on discrete fourier transform to improve the lifetime of underwater wireless sensor networks communications," in Proceedings of the ASEE Zone, vol. 1, pp. 1-5, Bridgeport, CT, USA, February 2014.

[4] C.-C. Lin, D.-J. Deng, and S.-B. Wang, "Extending the lifetime of dynamic underwater acoustic sensor networks using multipopulation harmony search algorithm," IEEE Sensors Journal, vol. 16, no. 11, pp. 4034-4042, 2016.

[5] S. Cheema, N. Javaid, S. A. Sheikh et al., "A balanced energy adaptive routing protocol with sector based node selection in underwater WSNs," in Proceedings of the NBIS, pp. 84-90, Ostrava, Czech Republic, September 2016.

[6] W. Y. Fu, D. S. Li, J. Chen et al., "Topology optimization based on balanced network energy and load in underwater acoustic sensor network," in Proceedings of the 6th WiCOM, pp. 1-5, Chengdu, China, December 2011.

[7] Z. Liao, D. Li, and J. Chen, "A network access mechanism for multihop underwater acoustic local area networks," IEEE Sensors Journal, vol. 16, no. 10, pp. 3914-3926, 2016.

[8] N. Goyal, M. Dave, and A. K. Verma, "Congestion control and load balancing for cluster based underwater wireless sensor networks," in Proceedings of the PDGC, pp. 462-467, Solan, India, March 2016.

[9] H. T. Zhang, W. D. Jiang, F. Tong et al., "Dynamic routing control based on energy balancing in underwater acoustic networks," Journal of Applied Sciences, vol. 23, no. 4, pp. 336-340, 2005.

[10] G. Z. Sun, C. J. Zhang, Z. Z. Lyu et al., "Routing protocols based on link-quality for underwater acoustic sensor networks," Mechatronics, vol. 15, no. 8, pp. 45-49, 2009.

[11] P. Wang, "Transmission loss threshold based routing design for underwater acoustic sensor networks," Electronic Science \& Technology, vol. 2, no. 1, pp. 108-113, 2015.

[12] X. Liu, Y. Li, C. H. Zhang et al., "Energy-balancing and delayrestricted routing protocol for underwater acoustic networks," Journal of Network New Media, vol. 2, no. 6, pp. 12-16, 2013.

[13] X. Li, Y. Q. Wang, and J. J. Zhou, “An energy-efficient clustering algorithm for underwater acoustic sensor networks," in Proceedings of the ICCECT, pp. 711-714, Liaoning, China, September 2012.

[14] K. Wang, Y. Z. Tian, and Y. H. Shi, "Energy balanced pressure routing protocol for underwater sensor networks," in Proceedings of the ICS, pp. 216-220, Chiayi, China, December 2016.

[15] P. Handa, Dr.B. S. Sohi, and N. Kumar, "Energy efficient hybrid routing protocol for underwater acoustic sensor network," in Proceedings of the ICEEOT, pp. 2573-2578, Chennai, India, March 2016.

[16] H. J. Shi and F. W. Liu, "Research of EBAP algorithm for ad Hoc underwater acoustic network," in Proceedings of the $3 \mathrm{rd}$ ICCC, pp. 464-468, Chengdu, China, December 2017.

[17] G. S. Yang, M. B. Xiao, E. Cheng et al., "Energy saving route finding mechanism in underwater acoustic sensor networks," Journal of Beijing University of Posts and Telecommunications, vol. 32, no. Sup, pp. 88-92, 2009.

[18] T. S. Hu and Y. S. Fei, "QELAR: a Q-learning-based energyefficient and lifetime-aware routing protocol for underwater sensor networks," in Proceedings of the IEEE IPCCC, pp. 247-255, Austin, TX, USA, December 2008.

[19] W. B. Zhang, "Design of virtual-path based energy efficiency routing for underwater acoustic sensor networks," M. S. thesis, Tsinghua University, Beijing, China, 2012.

[20] H. W. Liang, W. M. Chen, S. Li et al., "An ACO-based fair energy usage routing algorithm for wireless sensor networks," Pattern Recognition and Artificial Intelligence, vol. 20, no. 2, pp. 275-280, 2007.

[21] J. S. Abbasi, N. Javaid, S. Gull et al., "Balanced energy efficient rectangular routing protocol for underwater wireless sensor networks," in Proceedings of the IWCMC, pp. 1634-1640, Valencia, Spain, June 2017.

[22] M. F. Huang, K. Zhang, Z. W. Zeng et al., "An AUV-assisted data gathering scheme based on clustering and matrix completion for smart ocean," IEEE Internet of Things Journal, vol. 7, no. 10, 2020.

[23] G. J. Han, J. X. Du, C. Lin et al., "An energy-balanced trust cloud migration scheme for underwater acoustic sensor networks," IEEE Transcation Wireless Communications, vol. 19, no. 3, pp. 1636-1649, 2019.

[24] A. Majid, I. Azam, A. Waheed et al., "An energy efficient and balanced energy consumption cluster based routing protocol for underwater wireless sensor networks," in Proceedings of the 30th AINA, pp. 324-333, Crans-Montana, Switzerland, March 2016.

[25] S. Shen, A. D. Zhan, P. L. Yang et al., "Exploiting sink mobility to maximize lifetime in $3 \mathrm{~d}$ underwater sensor networks," in Proceedings of the IEEE ICC, pp. 1-7, Cape Town, South Africa, May 2010.

[26] J. M. J. Kartha and L. Jacob, "Lifetime enhancement in sparse underwater acoustic sensor networks using mobile elements," in Proceedings of the SPCOM, pp. 1-6, Bangalore, India, July 2012.

[27] W. Hyder, J. Poncela, and P. Otero, "Self-organized routing for radial underwater networks," in Proceedings of the INDIACom, pp. 918-922, New Delhi, India, March 2016.

[28] T. Liu, Q. L. Zhao, and L. B. Zhang, "Modified AODV routing protocol in underwater acoustic networks," in Proceedings of the ICEICT, pp. 191-194, Harbin, China, August 2016.

[29] N. Rajpoot and R. S. Kushwah, "An improved prophet routing protocol for underwater communication," in Proceedings of the ICCN, pp. 27-32, Gwalior, India, November 2015.

[30] R. F. W. Coates, Underwater Acoustic Systems, pp. 92-93, Macmillan, Basingstoke, UK, 1990.

[31] D. Xiao, L. P. Wei, G. Chen et al., "Inquiring flooding broadcast algorithm for underwater acoustic sensor self-organization networks," Journal of Applied Acoustics, vol. 34, no. 1, pp. 58-64, 2015.

[32] Y. X. Feng, D. Xiao, Y. Chen et al., "Relative self-positioning method for small-scale underwater acoustic network nodes without precise synchronization," Acta Acustica, vol. 45, no. 4, pp. 486-496, 2020. 